\title{
Análise das oportunidades de estimulação motora em ambientes domiciliares na região central do Rio Grande do Sul
}

CDD. 20.ed. 152.3

http://dx.doi.org/10.1590/1807-55092015000200279

\author{
Ellen Santos SOARES* \\ Fábio Saraiva FLORES ${ }^{* *}$ \\ Juliana Izabel KATZER ${ }^{* *}$ \\ Nadia Cristina VALENTINI ${ }^{* * *}$ \\ Sara Teresinha CORAZZA* \\ Fernando COPETTI*
}

*Centro de Educação Física e Desportos, Universidade Federal de Santa Maria.

**Escola Superior de Educação Física, Universidade Federal de Pelotas.

***Escola Superior de Educação Física, Universidade Federal do Rio Grande do Sul.

\section{Resumo}

Este estudo objetivou analisar a qualidade dos ambientes domiciliares na região central do Rio Grande do Sul para a ocorrência de oportunidades de estimulação motora. Fizeram parte do estudo 410 famílias de crianças com idades entre 18 e 42 meses. Para a avaliação foi utilizado o questionário "Affordances in the Home Enviroment for Motor Development Self-Report" - (AHEMD-SR) que aborda a qualidade e a quantidade de fatores no ambiente que são favoráveis à intensificação do desenvolvimento motor infantil. Foram utilizadas análises descritivas e os testes t para amostras independentes e ANOVA para comparação das médias. A análise dos dados evidenciou que as famílias com menor renda $(p=0,00)$ e cujos pais têm menor grau de instrução $(p=0,00)$ possuem lares que promovem menos oportunidades de estimulação motora às crianças. Os resultados menos satisfatórios foram em relação à provisão de materiais que estimulem o desenvolvimento da motricidade infantil no espaço externo das residências (entre 64,4\% e 65,1\% classificados como muito fraco). Conclui-se que, em grande parte das residências avaliadas, há uma carência na provisão de materiais que promovam a estimulação do desenvolvimento da motricidade infantil. 0 estudo dá fortes indícios que estas restrições podem ser decorrentes do baixo nível socioeconômico e grau de escolaridade das familias pesquisadas.

Palavras-chave: Habitação; Características da família; Fatores socioeconômicos; Desenvolvimento infantil.

\section{Introdução}

Pesquisas recentes ${ }^{1-4}$ indicam que fatores ambientais podem modificar o curso do desenvolvimento na infância, uma vez que esse processo depende da interação entre o indivíduo, o ambiente e a tarefa. Ambientes inadequados, inibidores ou pouco estimulantes podem repercutir de forma negativa no desenvolvimento da criança ${ }^{5-8}$.

Os primeiros anos de vida são extremamente importantes para o desenvolvimento da capacidade motora, sendo que os vínculos mais significantes, os principais cuidados e estímulos necessários ao crescimento e desenvolvimento, são fornecidos pela família 5 . O ambiente da casa familiar proporciona às crianças uma estimulação precoce e regular essencial na modelação do seu desenvolvimento9. Consequentemente, a estimulação por meio da disponibilidade de objetos, livros, jogos e brinquedos dentro de casa são indicadores críticos para a qualidade do ambiente ${ }^{10}$.

Neste contexto, a quantidade e qualidade das oportunidades de estimulação motora presentes nos domicílios têm um valor inestimável no espaçotempo de desenvolvimento da criança ${ }^{11}$. Estas oportunidades são oferecidas pelo ambiente que confere ao indivíduo desafios para a ação e contribuem para uma interaçáo dinâmica com o meio ${ }^{12}$, podendo ser potencializadas por objetos, eventos ou locais ${ }^{11}$.

A exploração do ambiente caracteriza-se como desencadeadora de diversas estratégias adaptativas que permitem ao ser humano a interaçáo com o meio. Esse fenômeno que faz referência à interação 
entre agente e ambiente é denominado "affordances"12. Estas são as diversas possibilidades de ação fornecidas pelo ambiente no qual o indivíduo está inserido, ou ainda, são as informaçóes disponíveis no ambiente que podem ser percebidas pelo indivíduo e construídas a partir da experiência, exploração e da percepção do agente em relação ao ambiente ${ }^{12}$. Nesse caso, espaços, materiais, brinquedos, jogos, variedades e práticas de interaçóes familiares, liberdade de movimentos e ação, constituem algumas das "affordances" presentes no dia a dia da criança ${ }^{13}$.

Além disto, juntamente com a organização estrutural do ambiente físico, é necessária a presença de indivíduos que promovam e auxiliem no processo de desenvolvimento. As formas de provisão das "affordances" motoras e, consequentemente, a qualidade dos estímulos fornecidos por estes indivíduos poderá depender de alguns fatores como o grau de escolaridade, o nível socioeconômico da família, ou ainda, a diferenciação entre os gêneros das crianças, visto que, os interesses, brincadeiras e as práticas motoras se diferenciam grandemente de um para outro ${ }^{5,14-17}$.

\section{Método}

Esta pesquisa caracteriza-se como descritiva e quantitativa ${ }^{21}$. O estudo foi aprovado pelo Comitê de Ética em Pesquisa da Universidade Federal de Santa Maria (número 0340.0.243.000-10).

\section{Amostra}

A amostra foi voluntária e intencional na qual fizeram parte famílias com crianças em idades entre $18 \mathrm{e}$ 42 meses (35,19 $\pm 8,20$ meses), sendo 198 meninas e 212 meninos, matriculadas em instituições públicas e privadas de educaçáo infantil cadastradas na Secretaria Municipal de Educação (SMED) e no Sindicato dos Professores do Ensino Privado do Rio Grande do Sul (SINPRO), da cidade de Santa Maria - RS.

De um total de 46 instituições cadastradas, 19 públicas e 27 privadas, 26 aceitaram participar do estudo, sendo 12 públicas e 14 privadas. Após o contato com as instituiçóes, foram identificadas 584 famílias com filhos entre 18 e 42 meses de idade e todas foram convidadas a participar da pesquisa. Destas 410 aceitaram participar e responder voluntariamente ao questionário.
Cabe ainda ressaltar que as crianças que vivem em países em desenvolvimento, como é o caso do Brasil, estão expostas a situaçôes de vulnerabilidade social decorrentes principalmente dos baixos níveis de instrução e condição econômica familiar e podem, consequentemente, apresentar atrasos em seu potencial de crescimento e desenvolvimento ${ }^{1,15,18}$. Considerando-se que o desenvolvimento motor apropriado está associado às experiências vivenciadas pela criança em um ambiente rico em incentivos e desafios ${ }^{2,16,19-20}$, é notória a necessidade de conhecer o perfil de uma população a fim de ampliar a compreensão sobre os fatores que influenciam nas interaçōes entre a criança, a família e o contexto ${ }^{1}$. Em vista disso, este estudo teve como objetivo analisar a qualidade dos ambientes domiciliares na regiáo central do Rio Grande do Sul para a ocorrência de oportunidades de estimulação motora. Especial atenção foi dada para a compreensão dos efeitos do sexo da criança, nível socioeconômico e escolaridade dos pais nas características de estimulação presentes nos ambientes investigados.

\section{Procedimentos}

Inicialmente entrou-se em contato com os responsáveis pela direção de cada uma das instituições de educação infantil e, posteriormente, foi feito contato com as famílias por meio de uma carta entregue pelos educadores. Após foram realizadas reuniōes nas quais foram explicados os objetivos e combinados os procedimentos adotados na pesquisa e todos os responsáveis pela tutela das crianças que concordaram em participar assinaram o Termo de Consentimento Livre e Esclarecido (TCLE) e receberam o questionário. Para aqueles que não puderam comparecer nas reunióes, os questionários foram enviados para as residências juntamente com uma carta explicativa e o TCLE, e respondidos por um adulto responsável pela criança, preferencialmente, aquele que mais a acompanhava em suas atividades diárias. Esse procedimento teve auxílio dos educadores das instituiçôes para entrega e recolhimento do material.

Para a análise das oportunidades na promoção do desenvolvimento motor do ambiente doméstico foi utilizado o questionário "Affordances in the Home 
Enviroment for Motor Development Self-Report" (AHEMD-SR) ${ }^{22-24}$. O questionário tem por objetivo avaliar o quanto o ambiente permite e promove potencialmente o desenvolvimento motor. Para tanto, aborda a qualidade e a quantidade de fatores no ambiente que são favoráveis à intensificação do desenvolvimento motor em crianças com idades entre 18-42 meses. Constitui-se por uma parte inicial sobre a identificação da criança e da família, e seguida por 67 questôes específicas sobre as oportunidades de estimulação presentes no ambiente. $\mathrm{O}$ nível socioeconômico é avaliado por meio da renda familiar mensal relatada a partir de uma classificação em seis grupos apresentados no instrumento (Menos de $\mathrm{R} \$ 1000 ; \mathrm{R} \$ 1000$ até $\mathrm{R} \$ 1500 ; \mathrm{R} \$ 1500$ até 2500; R\$2500 até R \$3500; R \$3500 até R \$5000; $\mathrm{R} \$ 5000$ ou mais). O nível de escolaridade dos pais é avaliado a partir de quatro classificaçóes (Ensino Fundamental, Ensino Médio, Curso Superior, Mestrado/ Doutorado), também sugeridas pelo instrumento.

Sua escala de classificação é subdividida em cinco categorias (Espaço Exterior, Espaço Interior, Variedade de Estimulação, Materiais de Motricidade Fina e Materiais de Motricidade Grossa). Para a avaliação da pontuação total são utilizadas três classificaçóes: "baixa" (até nove), "média" (de 10 a 16) e "alta" (de 17 a 20). Os resultados referentes as cinco categorias do AHEMD-SR são classificados em uma escala do tipo Likert, categorizada da seguinte forma: "muito fraco", "fraco", "bom" e "muito bom". Para gerar os resultados, as respostas de todas as questóes são inseridas em uma planilha que acompanha o questionário e então fornece automaticamente o cálculo da pontuação adequada de acordo com a idade do participante.

\section{Análise dos resultados}

Para a análise dos resultados, inicialmente os dados foram inseridos em uma planilha especificamente desenvolvida para o "software" Excel (AHEMD Calculador VPbeta1.5.xls) pelos autores do instrumento; a qual propicia o escore total, a classificação das oportunidades de estimulação em três categorias e sugestóes acerca dos resultados para cada indivíduo ${ }^{22-23}$. Os dados foram analisados no pacote estatístico SPSS, versão 14.0. A análise da distribuição dos dados foi feita através do teste de Kolmogorov-Smirnov, a partir do qual os dados foram classificados como paramétricos $(\mathrm{p}>0,05)$. Foram feitas análises descritivas e posteriormente foram aplicados os testes t de "student" para amostras independentes, para verificar a diferença das características do ambiente entre os sexos, e a análise de variância (ANOVA) One-Way com ajuste de Tukey para comparaçóes múltiplas, para verificar a diferença entre os fatores renda e escolaridade dos pais $^{25}$. Adotou-se o nível de significância de 5\%.

\section{Resultados}

A TABELA 1 apresenta a descrição dos resultados do AHEMD-SR total da amostra. A análise dos dados indica que a maior parcela $(60,97 \%)$ das residências avaliadas obteve classificação final "Média".

Na sequência, a TABELA 2 apresenta a descrição detalhada da distribuição dos resultados, em frequências, para cada uma das quatro classificaçóes das cinco categorias que compóem o AHEMD-SR. Os melhores escores foram observados no espaço interior das residências com $78 \%$ da amostra na classificação "Muito Bom". Em contrapartida, os escores mais baixos do estudo foram em relação à provisão de materiais de motricidade fina e grossa $(64,4 \%$ e $65,1 \%$, respectivamente), ambos com a maior parcela da amostra concentrada na classificação "Muito Fraco".

A TABELA 3 apresenta os resultados encontrados para o AHEMD-SR total de acordo com o nível de escolaridade dos pais. Nâo estão representados os dados de $4 \%$ dos pais e $2,8 \%$ das mães que não informaram a escolaridade. A análise de variância evidenciou que o grau de escolaridade foi significativamente diferente no AHEMD-SR total tanto para os pais $(\mathrm{f}=31,325$; $\mathrm{p}$ $=0,000)$, quanto para as mães $(f=32,831 ; p=0,000)$. Ao observar as médias é possível perceber que quanto mais alto o nível de escolaridade dos pais, mais alto foi o escore do AHEMD-SR total.

A TABELA 4 apresenta informações referentes ao nível socioeconômico da amostra, com uma classificação em cinco grupos de acordo com o rendimento mensal, conforme sugere o AHEMD-SR. Não foram informados os dados de 3,7\% das famílias que, por isso, não estão representados na tabela. A análise estatística através da comparação das médias revelou uma diferença significativa $(f=24,575$; $p=$ $0,000)$ a favor das famílias com maior rendimento mensal. Ao observar as médias referentes ao AHEMDSR total é possível notar que esses valores aumentam conforme aumenta o rendimento mensal da família. 
A TABELA 5 apresenta os resultados obtidos para cada item referente às "affordances" presentes no ambiente domiciliar. A análise da diferença entre sexos revelou que existe uma diferença significativa apenas nos três últimos itens avaliados.

TABELA 1 - Descrição dos resultados de acordo com a classificação do AHEMD-SR total.

\begin{tabular}{lcccc}
\hline Classificação & n (\%) & Mínimo - Máximo & Média (pontos) & Desvio padráo (pontos) \\
\hline Baixo & $141(34,39 \%)$ & $5-9$ & 7,99 & 1,10 \\
Médio & $250(60,97 \%)$ & $10-16$ & 11,98 & 1,79 \\
Alto & $19(4,63 \%)$ & $17-19$ & 17,68 & 0,74 \\
\hline
\end{tabular}

TABELA 2 - Distribuição de frequências e respectivo percentual dos valores relativos as cinco sub-escalas de avaliação do questionário.

\begin{tabular}{lcccc}
\hline Escalas & Muito fraco & Fraco & Bom & Muito bom \\
\hline Espaço exterior & $74(18,0 \%)$ & $125(30,5 \%)$ & $146(35,6 \%)$ & $65(15,9 \%)$ \\
Espaço interior & $31(7,6 \%)$ & $24(5,9 \%)$ & $34(8,3 \%)$ & $321(78,3 \%)$ \\
Variedade de estimulação & $74(18,0 \%)$ & $98(23,9 \%)$ & $90(22,0 \%)$ & $148(36,1 \%)$ \\
Materiais motricidade fina & $264(64,4 \%)$ & $89(21,7 \%)$ & $43(10,5 \%)$ & $14(3,4 \%)$ \\
Materiais motricidade grossa & $267(65,1 \%)$ & $82(20,0 \%)$ & $46(11,2 \%)$ & $15(3,7 \%)$ \\
\hline
\end{tabular}

TABELA 3 - Descrição dos dados referentes ao AHEMD-SR total de acordo com o nível de escolaridade dos pais.

* Significativo para $p<$ 0,05 (ANOVA).
* Significativo para $p<$ 0,05 (ANOVA).

\begin{tabular}{llcccc}
\hline Grupos & Nível de instruçáo & $\mathbf{n}$ & Mín-Máx (pontos) & Média \pm DP (pontos) & Significância \\
\hline \multirow{4}{*}{ Pai } & Fundamental & $136(32,5 \%)$ & $5-18$ & $9,73 \pm 2,19$ & $0,00^{*}$ \\
& Médio & $179(42,7 \%)$ & $5-19$ & $10,75 \pm 2,71$ & $0,00^{*}$ \\
& Curso superior & $75(17,9 \%)$ & $7-19$ & $13,00 \pm 2,80$ & 0,72 \\
& Mestrado/Doutorado & $12(2,9 \%)$ & $10-18$ & $13,83 \pm 3,09$ & 0,72 \\
& Fundamental & $105(25,1 \%)$ & $5-18$ & $9,66 \pm 2,44$ & $0,03^{*}$ \\
\multirow{4}{*}{ Mãe } & Médio & $204(48,7 \%)$ & $5-18$ & $10,49 \pm 2,56$ & $0,00^{*}$ \\
& Curso superior & $87(20,8 \%)$ & $5-19$ & $12,76 \pm 2,79$ & 0,10 \\
& Mestrado/Doutorado & $11(2,6 \%)$ & $12-18$ & $14,64 \pm 2,33$ & 0,10 \\
\hline
\end{tabular}

TABELA 4 - Descrição dos dados referentes ao AHEMD-SR total de acordo com o rendimento mensal das famílias.

\begin{tabular}{lcccc}
\hline Grupos & $\mathbf{n}$ & Mín-Máx (pontos) & Média \pm DP & Significância \\
\hline 1. Até R\$ 1.000 & $161(38,4 \%)$ & $5-19$ & $10,89 \pm 2,84$ & 0,96 \\
2. Entre R \$ 1.000 e R \$ 1.500 & $92(22,0 \%)$ & $5-18$ & $10,32 \pm 2,55$ & 0,28 \\
3. Entre R \$ 1.500 e R \$ 2.500 & $54(12,9 \%)$ & $7-18$ & $12,04 \pm 2,57$ & $0,00^{*}$ \\
4. Entre 2.500 e R \$ 3.500 & $31(7,4 \%)$ & $8-19$ & $12,48 \pm 2,85$ & $0,00^{*}$ \\
5. Entre R \$ 3.500 e R \$ 5.000 & $22(5,3 \%)$ & $8-19$ & $12,68 \pm 2,91$ & $0,00^{*}$ \\
6. Acima de R \$ 5.000 & $43(10,3)$ & $9-18$ & $13,35 \pm 2,44$ & $0,00^{*}$ \\
\hline
\end{tabular}

282 • Rev Bras Educ Fís Esporte, (São Paulo) 2015 Abr-Jun; 29(2):279-88 
TABELA 5 - Valores relativos aos itens avaliados no AHEMD-SR em relação ao sexo da criança.

\begin{tabular}{|c|c|c|c|c|c|c|}
\hline Variáveis & Sexo & Média & Desvio padráo & $\mathbf{t}$ & $\mathbf{p}$ & \multirow{3}{*}{$\begin{array}{l}\text { * Significativo para } p< \\
0,05 \text { (Teste t para amos- } \\
\text { tras independentes). }\end{array}$} \\
\hline \multirow[t]{2}{*}{ Estimulação } & Masculino & 3,75 & 1,34 & 0,492 & 0,623 & \\
\hline & Feminino & 3,69 & 1,45 & & & \\
\hline \multirow[t]{2}{*}{ Liberdade } & Masculino & 2,31 & 0,67 & 0,49 & 0,62 & \\
\hline & Feminino & 2,27 & 0,70 & & & \\
\hline \multirow[t]{2}{*}{ Incentivo } & Masculino & 2,32 & 0,82 & $-0,97$ & 0,32 & \\
\hline & Feminino & 2,40 & 0,79 & & & \\
\hline \multirow[t]{2}{*}{ Atividades diárias } & Masculino & 4,61 & 1,10 & 0,60 & 0,54 & \\
\hline & Feminino & 4,54 & 1,16 & & & \\
\hline \multirow[t]{2}{*}{ Bonecos faz de conta } & Masculino & 16,20 & 6,19 & $-1,61$ & 0,10 & \\
\hline & Feminino & 17,15 & 5,66 & & & \\
\hline \multirow[t]{2}{*}{ "Puzzles” } & Masculino & 5,17 & 4,48 & 1,53 & 0,12 & \\
\hline & Feminino & 4,48 & 4,69 & & & \\
\hline \multirow[t]{2}{*}{ Jogos } & Masculino & 2,13 & 2,37 & 0,82 & 0,41 & \\
\hline & Feminino & 1,93 & 2,49 & & & \\
\hline \multirow[t]{2}{*}{ Materiais de construção } & Masculino & 1,82 & 1,95 & 1,67 & 0,09 & \\
\hline & Feminino & 1,51 & 1,86 & & & \\
\hline \multirow[t]{2}{*}{ Brinquedos educacionais } & Masculino & 8,43 & 4,58 & 0,14 & 0,88 & \\
\hline & Feminino & 8,36 & 4,38 & & & \\
\hline \multirow[t]{2}{*}{ Materiais musicais } & Masculino & 5,14 & 3,57 & 1,53 & 0,12 & \\
\hline & Feminino & 4,61 & 3,47 & & & \\
\hline \multirow[t]{2}{*}{ Materiais manipulativos } & Masculino & 2,34 & 1,53 & $3,77^{*}$ & 0,00 & \\
\hline & Feminino & 1,80 & 1,38 & & & \\
\hline \multirow[t]{2}{*}{ Materiais locomotores } & Masculino & 4,25 & 2,56 & $3,32^{*}$ & 0,00 & \\
\hline & Feminino & 3,44 & 2,36 & & & \\
\hline \multirow[t]{2}{*}{ Materiais de exploração global } & Masculino & 0,81 & 0,94 & $-2,37^{*}$ & 0,01 & \\
\hline & Feminino & 1,06 & 1,17 & & & \\
\hline
\end{tabular}

\section{Discussão}

O principal propósito do estudo foi analisar a qualidade dos ambientes familiares na região central do Rio Grande do Sul para a ocorrência de "affordances", com ênfase na compreensão dos efeitos do sexo da criança, nível sócioeconômico e escolaridade dos pais nas características de estimulação presentes nos ambientes investigados.

Os resultados gerais permitem observar que o ambiente familiar foi classificado predominantemente como médio na presente amostra. RodRIGUES e GABBARD ${ }^{17}$ descrevem que uma residência classificada como média providencia apenas oportunidades razoáveis para o desenvolvimento motor da criança. Nobre et al. ${ }^{16}$ alertam para o fato de que a insuficiência de oportunidade de estímulos ambientais poderá comprometer o bom desenvolvimento motor das crianças, visto que, a disponibilidade de materiais, objetos e jogos estimulantes são importantes preditores do desenvolvimento.

Observando-se os resultados quanto ao espaço exterior e interior da residência, a variedade de estimulação e quantidade de brinquedos de motricidade fina e grossa, pode-se inferir que em grande parte das residências as oportunidades de estimulação motoras oferecidas não parecem ser suficientes para promover o desenvolvimento motor adequado das crianças. 
Apenas 4,63\% dos ambientes receberam classificação "alta", o que seria o resultado satisfatório e desejado. Fica evidente também que os ambientes domiciliares possuem uma fragilidade quanto aos materiais disponíveis para utilização das crianças, fato que implica diretamente no desenvolvimento da motricidade na infância, o que é bastante preocupante.

Embora os resultados do presente estudo representem as características ambientais das residências da cidade de Santa Maria, regiâo central do RS, outros estudos desenvolvidos em regiōes diferentes do Brasil têm demonstrado que essas características parecem não diferir muito de região para regiāo. Um estudo ${ }^{4}$ feito na cidade de Florianópolis - SC, obteve resultados similares. A avaliação da qualidade do ambiente domiciliar de 20 famílias revelou que as oportunidades que os domicílios oferecem para as crianças desenvolverem as motricidades fina e grossa também não estão sendo suficientes, visto que $75 \%$ e $65 \%$, respectivamente, das casas avaliadas obtiveram classificação "fraca" ou "muito fraca”. Os melhores resultados de Florianópolis, da mesma maneira que os deste estudo, foram encontrados nas subescalas espaço interior $(80 \%)$ e variedade de estimulação (45\%), classificados como "muito bom".

No Ceará, resultados semelhantes foram encontrados em um estudo ${ }^{16}$ feito com 128 famílias. Os resultados também foram considerados bastante preocupantes, visto que, dentre os ambientes residenciais avaliados, a maior parte dos espaços físicos externos receberam classificação "fraca" e "muito fraca" no AHEMD-SR, o que, segundo os autores, demonstra uma prevalência da inadequação das estruturas arquitetônicas das residências para favorecer o desenvolvimento motor. Além disso, os resultados mais preocupantes, do mesmo modo que no presente estudo e no estudo de Florianópolis, foram relacionados aos materiais que promovem o desenvolvimento da motricidade, pois evidenciaram a inexistência de materiais em quantidades suficientes para um pleno desenvolvimento da motricidade grossa e fina das crianças.

Nesse sentido, outro fator que chama atenção é o fato de o espaço interno da maioria dos lares ter recebido classificação "boa", o que indica que pode existir uma preocupação maior por parte dos pais em relação à organização das estruturas internas domiciliares, como a disposição dos móveis e a quantidade e acomodação dos espaços, por exemplo, do que em adquirir brinquedos, jogos e materiais que oportunizem condiçóes de experimentaçáo motora.

Estudo realizado em São Paulo por MARIA-MenGEL e LINHAREs $^{26}$, no qual utilizaram o Inventário HOME para Observação e Medida do Ambiente, constatou que $74 \%$ das crianças apresentaram um nível de estimulação no ambiente familiar classificado como "médio". Verificou-se que as subescalas organização do ambiente físico em geral e oportunidades para variar o estímulo do cotidiano obtiveram pontuaçóes altas, enquanto a provisão de materiais apropriados à idade da criança obteve escores mais baixos. De acordo com as autoras, isso significa que em algumas famílias, não havia brinquedos disponíveis para a criança.

Cabe ainda ressaltar que os resultados observados no nosso estudo não refletem apenas a realidade das residências localizadas no Brasil. Resultados similares utilizando o AHEMD-SR foram encontrados no $\operatorname{Iran}^{10}$ em um estudo feito com 350 famílias com crianças de idades entre 18 e 42 meses. A análise das médias obtidas nas cinco subescalas revelou que apenas a variedade de estimulação e o ambiente interior das residências receberam classificação "Bom". O espaço exterior classificou-se como "fraco" e os materiais de motricidade fina e grossa também receberam classificação "muito fraco", caracterizando, da mesma maneira, uma situação preocupante.

Em vista de todos estes resultados expostos é possível perceber uma situação preocupante em relação à qualidade dos ambientes domiciliares. Nesse caso, alguns fatores referentes às características familiares também devem ser levados em consideração, visto que podem influenciar nas decisóes familiares em relação à estruturação das residências. Ao observar as médias é possível perceber que quanto mais alto o nível de escolaridade dos pais, mais alto foi o escore do AHEMD-SR total.

O grau de instrução dos pais também foi um fator significativo em um estudo feito por MARTINs et al. ${ }^{7}$, que buscou investigar os fatores de risco associados à qualidade do ambiente de 634 crianças da cidade de Pelotas - RS. Foi encontrada uma associaçáo significativa entre o nível de escolaridade materna, o nível de escolaridade paterna e a qualidade do ambiente, sendo que, a prevalência de ambientes negativos aumentava à medida que diminuíam as categorias de escolaridade (teste de tendência linear $<0,01$ ). Os autores supóem que os pais com escolaridade maior provavelmente tiveram mais acesso a informaçóes sobre desenvolvimento infantil e, por isso, interagem melhor com seus filhos, respondem adequadamente às suas solicitaçôes e podem prover melhores condiçôes físicas e emocionais para o seu desenvolvimento.

Outro estudo que corrobora com o presente foi desenvolvido por ANDRADE et al. ${ }^{5}$, que avaliaram o ambiente familiar de 350 crianças com idades entre 17 
e 42 meses em Salvador - BA e também encontraram uma associação significativa $(\mathrm{p}<0,001)$ entre o nível de escolaridade materna e a qualidade da estimulação ambiental recebida pela criança no ambiente familiar.

Investigando o nível de escolaridade e de renda como fatores de risco, Maria-Mengel e Linhares ${ }^{26}$ em um estudo descritivo realizado com 120 crianças de seis a 44 meses identificaram que os cuidadores das crianças apresentavam baixo nível de escolaridade (em torno de quatro anos de estudo) e tinham renda familiar mediana inferior a dois salários mínimos vigentes, o que caracterizou que as crianças possuíam contextos de desenvolvimento com múltiplos riscos psicossociais. As autoras concluíram que quanto maior o nível de escolaridade dos pais, maior poderá ser a renda familiar e, consequentemente, melhores as possibilidades de oferecer estímulos adequados para favorecer o desenvolvimento da criança.

Ao observar as médias referentes ao AHEMD-SR total é possível notar que esses valores aumentam conforme aumenta o rendimento mensal da família, o que confirma que o nível socioeconômico é também um fator determinante na qualidade do ambiente. Corroborando estes resultados, MARTins et al. ${ }^{7}$, avaliaram a qualidade dos ambientes de crianças e identificaram que a qualidade desses estava diretamente associada com o nível socioeconômico das famílias. Verificaram que à medida que diminuíam os níveis de renda, aumentava o percentual de ambientes negativos. A principal categoria de renda familiar situou-se entre 3,1 e 6, salários-mínimos (30,50\%).

Nobre et al. ${ }^{16}$ afirmam que o nível econômico é determinante nas melhores condições de escolaridade dos tutores, o que influencia diretamente nas oportunidades facilitadoras para um efetivo desenvolvimento motor das crianças. Essa afirmação é baseada no registro de diferenças significativas a favor dos pais $(\mathrm{p}=0,001)$ e das mães $(\mathrm{p}=0,000)$ de lares com maior renda no Ceará. Além disso, estudos têm comprovado a associação do nível socioeconômico com diversos fatores de risco para o desenvolvimento infantil, os quais se relacionam diretamente com a qualidade do ambiente. Pilz e SchermanN ${ }^{6}$, constataram em um estudo feito com 197 crianças de zero a seis anos do município de Canoas - RS, que a renda familiar foi o fator que demonstrou maior associação com a suspeita de atraso no desenvolvimento neuropsicomotor. Crianças de famílias com renda de até um salário mínimo mostraram uma probabilidade 9,3 vezes maior de apresentarem suspeita de atraso do que crianças de família com renda maior do que três salários mínimos.
Também relacionando o nível socioeconômico com atraso no desenvolvimento, HALPERN et al..$^{27}$, avaliaram o desenvolvimento neuropsicomotor de 1.362 crianças com 12 meses de idade da cidade de Pelotas - RS, as quais apresentaram teste sugestivo de atraso no desenvolvimento associado com a renda familiar. $\mathrm{O}$ percentual de atraso diminuiu conforme o aumento da renda da família e foi duas vezes mais frequente entre as crianças de famílias mais pobres do que entre as de melhor situação sócioeconômica $(\mathrm{p}<0,001)$.

Foram observadas diferenças entre os sexos, porém, ficaram restritas às ofertas de materiais manipulativos, locomotores e materiais de exploração global. A literatura sugere que de acordo com o gênero dos filhos, as mães descrevem brincadeiras e tipos de objetos diferentes, estabelecendo normas específicas que determinam os objetos a serem utilizados por uns e náo por outros e as brincadeiras típicas de cada sexo $^{28}$. Rodrigues e GABBARD ${ }^{17}$ afirmam que as crianças desde muito cedo diferenciam grandemente em suas preferências e interesses por brincadeiras e práticas motoras de acordo com o gênero a que pertencem. Os autores exemplificam que desde os nove meses de idade as crianças já demonstram preferência por brinquedos característicos ao seu gênero: meninos escolhem carros, bolas, trens, volantes e cubos, enquanto meninas preferem bonecas, panelas, vassourinhas, ou aparelhos de cozinha. Entretanto, os resultados no presente estudo evidenciam que estas diferenças, na faixa etária estudada, ainda não emergem em todas as dimensóes da rotina da criança. Futuras pesquisas devem considerar investigar estes fatores com especial atenção, visto que os resultados de pesquisa são contraditórios.

A infância é a etapa mais significante em direção à maturidade para a vida adulta e, portanto, é imprescindível garantir que esse período traga condiçóes propícias e pertinentes para a evolução e desenvolvimento. Os resultados encontrados neste estudo revelam uma situação preocupante, uma vez que, indicam que os estímulos ambientais que são oportunizados pelas famílias às crianças em grande parte das residências avaliadas não estão sendo suficientes para uma adequada promoçáo do desenvolvimento motor infantil.

Como conclusão do presente estudo, vários aspectos chamam a atenção para as oportunidades oferecidas às crianças. A carência na provisão de materiais dentro das residências que estimulem o desenvolvimento da motricidade infantil com certeza é um fator que merece atenção. Além disso, escores baixos também foram evidenciados em relação ao 
espaço externo das moradias, o que também possui influencia direta nas oportunidades de vivência e exploração dos movimentos. O estudo dá fortes indícios que estas restrições podem ser decorrentes do nível socioeconômico e grau de escolaridade das famílias pesquisadas. $\mathrm{O}$ estudo evidenciou que as famílias com menor renda mensal e cujos pais têm um menor grau de instrução possuem lares que promovem menos oportunidades de estimulaçáo motora às crianças.

Os resultados ressaltam a importância da avaliação dos ambientes domiciliares e da identificaçáo dos riscos aos quais as crianças estão expostas. Essas informaçóes podem ser estendidas aos pais, administradores e educadores de instituiçóes infantis para que os mesmos possam proporcionar às crianças oportunidades de estimulação que auxiliem no seu desenvolvimento. A melhor qualidade e quantidade de equipamentos, brinquedos, a disponibilização de espaços físicos mais amplos podem oportunizar as crianças condiçôes otimizadas de explorar, vivenciar, aprender e se desenvolver.

Esse trabalho ressalta a importância do ambiente domiciliar para a promoção e proteção do desenvolvimento infantil. Entretanto, faz-se necessário ressaltar a relevância da avaliação dos demais contextos em que a criança está inserida frequentemente, bem como o seu desenvolvimento motor para que possam ser obtidas informações mais fidedignas e completas sobre o papel dos contextos no desenvolvimento infantil. Nesse sentido, as limitaçôes desse estudo referem-se à necessidade de dar continuidade e ampliar as avaliaçóes de ambiente e desenvolvimento da criança, para que esses dados possam ser utilizados de forma eficaz e auxiliem na implementação de políticas públicas de atenção à infância.

\begin{abstract}
Analysis of the opportunities of motor stimulation in home environment in the central region of Rio Grande do Sul

This study aimed to analyze the quality of home environments in the central region of Rio Grande do Sul to the occurrence of motor stimulation opportunities. Participants were 410 children aged between 18 and 42 months. For the assessment of home environments we used the Affordances in the Home Environment for Motor Development Self-Report - (AHEMD-SR). This instrument addresses the quality and quantity of factors of home environment that can enhance children's motor development. Descriptive analysis, $t$ tests for independent samples and ANOVA were used to compare means. Data analysis showed that households with lower wages $(p=0.00)$ and whose parents have lower education $(p=0.00)$, have home environments that provide fewer opportunities for motor stimulation to children. The results were less satisfactory in relation to the provision of materials that encourage and stimulate children's motor development, on the external space of the residences (between $64,4 \%$ and $65,1 \%$ is classified as a very poor). It is concluded that there is a shortage in supply of materials that promote the stimulation of motor development in children, in most residences evaluated. The study provides strong evidence that these restrictions result from low socioeconomic and educational level of the families surveyed.
\end{abstract}

KeY Words: Housing; Family characteristics; Socioeconomic factors; Child development.

\title{
Referências
}

1. Zajonz R, Muller AB, Valentine NC. A influência de fatores ambientais no desempenho motor e social de crianças da periferia de Porto Alegre. Rev Educ Fís/UEM. 2008;19:159-71.

2. Santos DCC, Tolocka RE, Carvalho J, Heringer LRC, Almeida CM, Miquelote CM. Desempenho motor grosso e sua associação com fatores neonatais, familiares e de exposição à creche em crianças até três anos de idade. Rev Bras Fisioter. 2009;13:173-9.

3. Baltieri L, Santos DCC, Gibim NC, Souza CT, Batistela ACT, Tolocka RE. Desempenho motor de lactentes frequentadores de berçários em creches públicas. Rev Paul Pediatr. 2010;28:283-9. 
4. Nazario PF. Desempenho motor e as affordances do contexto [dissertação]. Florianópolis (SC): Universidade do Estado de Santa Catarina, Centro de Ciências da Saúde e do Esporte; 2011.

5. Andrade AS, Santos DN, Bastos AC, Pedromônico MRM, Almeida-Filho N, Barreto M. Ambiente familiar e desenvolvimento cognitivo infantil: uma abordagem epidemiológica. Rev Saúde Públ. 2005;39:606-11.

6. Pilz EML, Schermann LB. Determinantes biológicos e ambientais no desenvolvimento neuropsicomotor em uma amostra de crianças de Canoas/RS. Ciênc Saúde Colet. 2007;12:181-90.

7. Martins MFD, Costa JSD, Saforcada ET, Cunha MDC. Qualidade do ambiente e fatores associados: um estudo em crianças de Pelotas, Rio Grande do Sul, Brasil. Cad Saúde Públ. 2004;20:710-8.

8. Spessato BC, Valentini NC, Krebs RJ, Berleze A. Educação infantil e intervenção motora: um olhar a partir da teoria bioecológica de Bronfenbrenner. Movimento. 2009;15:147-73.

9. Bronfenbrenner U. A ecologia do desenvolvimento humano: experimentos naturais e planejados. Porto Alegre: Artes Médicas; 1996.

10. Haydari A, Askari P, Nezhad MZ. Relationship between affordances in the home environment and motor development in children age 18-42 months. J Soc Sci. 2009;5:319-28.

11. Rodrigues LP, Gabbard C. Avaliação das oportunidades de estimulação motora presentes na casa familiar: projecto affordances in the home environment for motor development. In: Barreiros J, Cordovil R, Carvalheira S, editores. Desenvolvimento motor da criança. Lisboa: Ediçôes FMH; 2007. p.51-60.

12. Gibson E. Exploratory behavior in the development of perceiving, acting, and the acquiring of knowledge. Ann Rev Psychol. 1988;39:1-42.

13. Caçola P, Gabbard C, Santos DCC, Batistela ACT. Development of the affordances in the home environment for motor development-infant scale. Pediatr Int. 2011;53:820-5.

14. Andraca I, Pino P, Parra A, Rivera F, Castillo M. Risck factors for psychomotor development among infants born under optimal biologicals conditions. Rev Saúde Públ. 1998;32:138-47.

15. Mancini MC, Megale L, Brandão MB, Melo APP, Sampaio RF. Efeito moderador do risco social na relaçáo entre risco biológico e desempenho funcional infantil. Rev Bras Saúde Mater Infant. 2004;4:25-34.

16. Nobre FSS, Costa ClA, Oliveira DL, Cabral DA, Nobre GC, Caçola P. Análise das oportunidades para o desenvolvimento motor (affordances) em ambientes domésticos no Ceará - Brasil. Rev Bras Crescimento Desenvolv Hum. 2009;19:9-18.

17. Rodrigues LP, Gabbard C. A invariância do gênero na estrutura multidimensional do AHEMD (affordances in the home environment for motor development). In: Catela D, Barreiros J, editores. Estudos em desenvolvimento motor da criança. Rio Maior: Edições ESDRRM; 2008. p.151-8.

18. Halpern R, Giugliani ERJ, Victora CG, Barros FC, Horta BL. Fatores de risco para suspeita de atraso no desenvolvimento neuropsicomotor aos 12 meses de vida. J Pediatr. 2000;76:421-8.

19. Almeida CS, Valentini NC, Lemos CXG. A influência de um programa de intervençáo motora no desenvolvimento de bebês no terceiro trimestre de vida em creches para a populaçáo de baixa renda. Temas Desenvolv. 2005;14:40-8.

20. Silva OPV. A importância da família no desenvolvimento do bebê prematuro. Psicol Teor Prát. 2002;4:15-24.

21. Thomas JR, Nelson JK, Silverman SJ. Métodos de pesquisa em atividade física. 5a ed. Porto Alegre: Artmed; 2007.

22. Rodrigues LP. Development and validation of the AHEMD-SR (affordances in the home environment for motor development-self report) [doctoral dissertation]. Brazos County (TX): A\&M University; 2005.

23. Rodrigues LP, Saraiva L, Gabbard C. Development and structural validation of an inventory for assessing affordances in the home environment for motor development. Res Q Exerc Sport. 2005;76:140-8.

24. Gabbard C, Caçola P, Rodrigues LP. A new inventory for assessing affordances in the home environment for motor development (AHEMD-SR). Early Child Educ J. 2008;36:5-9.

25. Barros MVG, Reis RS, Hallal PC, Florindo AA, Farias Júnior JC. Análise de dados em saúde. 3a ed. Londrina: Midiograf; 2012.

26. Maria-Mengel MRS, Linhares MBM. Fatores de risco para problemas de desenvolvimento infantil. Rev Latino-Am Enfermagem. 2007;15:837-42.

27. Halpern R, Barros FC, Victora CG, Horta BL. Desenvolvimento neuropsicomotor aos 12 meses de idade em uma coorte de base populacional no sul do Brasil: diferenciais conforme peso ao nascer e renda familiar. Cad Saúde Públ. $1996 ; 12: 1-42$.

28. Conti L, Sperb TM. O brinquedo de pré-escolares: um espaço de ressignificaçấo cultural. Psic Teor Pesq. 2001;17:59-67. 
Soares ES, et al.

Ellen Santos Sc

Centro de Educação Física e Desportos

Universidade Federal de Santa Maria

Av. Roraima, 1000 - Cidade Universitária - Prédio 51

97105-900 - Santa Maria - RS - BRASIL

e-mail: ellensoa@gmail.com
Recebido para publicação: 27/03/2013

Revisado: 14/08/2014

Aceito: 19/08/2014

288 • Rev Bras Educ Fís Esporte, (São Paulo) 2015 Abr-Jun; 29(2):279-88 